\title{
Buffalo and Maslow's hammer
}

Abraham H Maslow once said, "I suppose it is tempting, if the only tool you have is a hammer, to treat everything as if it were a nail". Kathleen Alexander and her coauthors describe a game scout's unsurprising finding in Botswana's Okavango Delta, and go on to consider the statistical equivalent of that famous hammer!

$\mathrm{T}_{1}^{\mathrm{k}}$ he Okavango Delta in Botswana, Africa, is one of the largest inland deltas in the world, a sprawling oasis teeming with wildlife in an otherwise water-scarce country. I was working for Botswana's Department of Wildlife and National Parks as the Senior Wildlife Veterinarian responsible for providing wildlife health services. We were doing a buffalo (Syncerus caffer) capture, to identify the population's movement dynamics and health status. Helicopter time was expensive, so we had developed a well-greased capture machine. There were only a few of us - the pilot, a biologist, and me, together with one of the game scouts from my unit. While I worked with the other team members on each animal's front end (taking blood samples, radio collaring, and ear tagging), the game scout conducted rectal examinations on captured buffalo cows to determine their pregnancy status. We would then switch business ends and I would repeat the rectal exam and double-check his findings.

That morning, as the mist lifted, we had just finished processing a captured animal in a dense palm island, working with a novice game scout. I never liked palm islands, because you're often unable to see three meters ahead of you. The helicopter is off in the distance and there is the continual, nagging thought that something might be watching you and is just seconds away from eating or maiming you - neither of which are attractive options. We quickly finished up with the animal, not saying a word. Once done, I injected the animal with the anesthetic's reversal agent and we ran to the helicopter, the pilot pulling us off the ground as we climbed into our seats. I then attended to our data sheet, trying to complete it before arriving at the next capture site. My game scout leaned over, tapped my shoulder (the noise of the helicopter was deafening), and screamed into his headset microphone, "Dr Alexander, it was empty!" - referring to the pregnancy status of the last animal, pleased with his newly applied diagnostic skills. I shouted back "That's great, because it was a bull!" With the helicopter full of men (aside from myself), there was great consternation at what this poor animal had suffered and obvious pontification at the clear consequences of gender mislabeling. Although anxious to communicate more about the indignities suffered by the female sex, I realized that I didn't have a sympathetic audience. They were simply worried that a member of the male species had suffered an indignity normally reserved for the female species. But that's another story... and not really the point of this story.

The game scout was looking for data (pregnancy status) in a place and way most people would not (rectal examination of a bull). The game scout did not have a preconceived notion on the subject and was open to possibility or perhaps blind to circumstances. In this example, the game scout was simply wrong in his selected approach. But this made my coauthors and I think about how we apply ourselves in the process of scientific discovery. How does our selection and use of scientific approaches, including statistical tools, impose limits or open doors to novel discoveries? Are we using the most appropriate tools or simply those that we're most familiar with? Are we amenable to trying new approaches (like the game scout, however inappropriately or unknowingly) in addition to the old ones? This is important because, ultimately, it is our statistical evaluation of the data that will determine what we are able to correctly infer from our research.

Three philosophically different paradigms are currently used in ecology to make statistical inference and come to conclusions about the meaning of collected data: null hypothesis testing (NHT), information-theoretic (I-T), and Bayesian inference (BI). Each has particular strengths but also some inherent weaknesses (WebTable 1). Taken together, these three approaches to inference offer a much richer toolset to ecologists today than ever before. Often, however, newer approaches (I-T, BI) are ignored as being too complex, and we carry on using our old tools without considering the advantages of the newer tools. Alternatively, the newer techniques become the latest craze, to the detriment of the older tools. Our goal here is to articulate what each tool has to offer and to encourage the understanding that scientific discovery will be enhanced by harnessing the strengths of all available tools - as determined by the questions at hand and the type of data generated by the research design. We invariably call for "eclecticism" (for the definition of this and other specialist terms, as well as suggestions for further reading, see WebPanels 1 and 2) in statistical education and applications in ecological research.

The case for eclecticism has been made before and resisted; key objections on either side of the debate have been raised and counter arguments provided. But ultimately, each statistical approach has its place in scientific analyses, and while we may not always use these tools properly (Dennis 1996; Anderson and Burnham 2002; Stephens et al. 2005), it is not a sufficient argument for the exclusion of any particular technique. Indeed, we see that a certain level of eclecticism characterizes the practice of all inferential paradigms and the applications they support. For example, a very common practice is the use of I-T and BI techniques in model selection and NHT in the evaluation of individual parameters retained in a model (Whittingham et al. 2006). A subtle form of this kind of eclecticism is the construction of a $(100-\alpha) \%$ confidence interval for parameter estimates when models are selected or averaged by $\mathrm{I}-\mathrm{T}$ techniques. A $95 \%$ confidence interval is the mathematical inversion of a null hypothesis test, with the probability of wrongly rejecting a true null hypothesis, com- 
monly referred to as a Type I error and symbolized by $\alpha$, set at 5\% (Casella and Berger 2002). Here are some examples to help illustrate the use of multiple tools, both new and old, in the statistical toolbox. While we concentrate on spatial applications available in distribution modeling and disease ecology, trends are similar in other ecological subdisciplines.

Various analytical approaches in ecology illustrate the use of, and need for, eclecticism. Modeling species' ranges demonstrates the integration of statistical approaches from all three paradigms to explore complex datasets. These modeling approaches estimate the environmental conditions and corresponding geography that sustain a species, often testing hypotheses rooted in ecological niche theory. Non-random relationships between species occurrence data and environmental variables are evaluated by these approaches, either through pattern matching with post hoc statistical evaluation or through statistical relationships (Blackburn 2010). Ecological niche, species distribution, and Bayesian habitat suitability models have been applied across many taxa to test various ecological concepts, such as niche shifting, differentiation, conservatism, and niche theories (eg Grinnellian versus Eltonian). Specific to disease ecology, distribution and niche models have been used to estimate the distribution of vectors, hosts, and pathogens, and more recently to estimate human populations at risk.

For example, the genetic algorithm for rule set prediction - a widely used niche modeling tool - employs a heuristic pattern matching approach that relies on evolutionary concepts (such as cross-over, insertions, and deletions) to modify rules (logic if/then statements) that relate variable conditions (eg temperature ranges) to point occurrences (known $x$ and $y$ coordinates of the target species being modeled). Other popular modeling approaches, such as MaxEnt, also rely on a maximum entropy distribution to predict species' distributions, whereas the nonlinear discriminant analysis and ecological niche factor analysis approaches fit uncorrelated factors (related to some quantitative characteristic of the environment) to the presence or absence of a species. Various accuracy and sensitivity metrics are available, representing several NHT (eg binomial tests, area under the curve, kappa statistic) and I-T (Akaike information criterion) techniques. Thus, model outcomes provide predictions of a species' range (presence/absence), and statistical applications from the paradigms are used to evaluate the accuracy of these predictions.

Spatial clustering statistics are increasingly applied in spatial ecology and epidemiological studies. These tests greatly improve our ability to evaluate incidence data and detect spatial variation, identifying hotspots of ecological phenomena (such as disease outbreaks). As with the examples above, many clustering approaches integrate multiple statistical paradigms in their application. For example, Bayes empirical smoothing (BES) and spatially weighted versions of BES provide a means of reducing variance between neighboring areal units where either low case numbers or population estimates can inflate prevalence rates in rural areas. For many infectious diseases particularly zoonoses - cases are usually low and detection limited, creating a need for such techniques. These smoothed surfaces can be linked with cluster analyses, where the cluster sta-

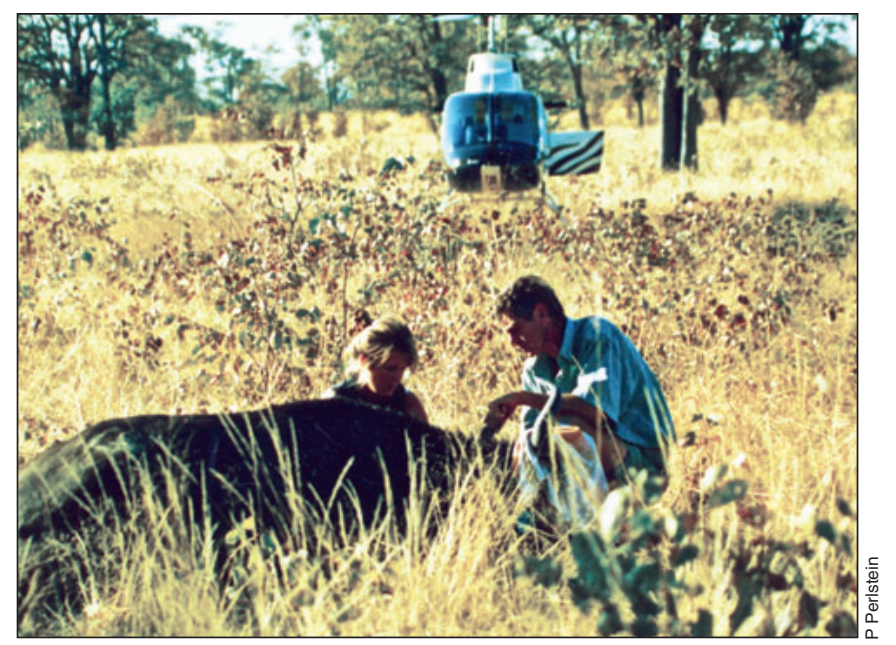

Figure 1. Sampling a captured buffalo in Botswana.

tistic incorporates a traditional hypothesis test, defining clusters with a probability cutoff, while the Bayesian approach is used to meet the assumptions of the NHT. These approaches can reduce variation across a spatial dataset and identify otherwise undetected spatial clustering of infectious disease.

A newer "landscape phylodynamics" approach - linking spatiotemporal analyses with the pathogen's phylogeographic attributes in a BI framework - is very useful in zoonotic studies, where disease movement is the product of coupled animal and human movement behaviors. In all of these examples, we see the exciting horizon of tool evolution and the incorporation of both new and old tools in statistical techniques, allowing us to expand our understanding of ecological phenomena. The story of the game scout thus serves as a reminder to ecologists of the importance of keeping an open mind about our approaches and to be vigilant in our efforts to understand advances in the toolbox - to engage eclecticism in this great age.

\section{References}

Anderson DR and Burnham KP. 2002. Avoiding pitfalls when using information-theoretic methods. J Wildlife Manage 66: 912-18.

Blackburn JK. 2010. Integrating geographic information systems and ecological niche modeling into disease ecology: a case study of Bacillus anthracis in the United States and Mexico. Emerg Endemic Pathog; doi:10.1007/978-90-481-9637-1_7.

Casella G and Berger RL. 2002. Statistical inference. Pacific Grove, CA: Duxbury Press.

Dennis B. 1996. Discussion: should ecologists become Bayesians? Ecol Appl 6: 1095-1103.

Stephens PA, Buskirk SW, Hayward GD, and Del Rio CM. 2005. Information theory and hypothesis testing: a call for pluralism. J Appl Ecol 42: 4-12.

Whittingham MJ, Stephens PA, Bradbury RB, and Freckleton RP. 2006. Why do we still use stepwise modelling in ecology and behaviour? J Animal Ecol 75: 1182-89.

\section{Kathleen A Alexander ${ }^{1 *}$, Jason K Blackburn ${ }^{2}$, and Emmanuel A Frimpong ${ }^{1}$ ${ }^{1}$ Department of Fish and Wildlife Conservation, Virginia Tech, Blacksburg,VA*(kathyalx@vt.edu); ${ }^{2}$ Emerging Pathogens Institute and Department of Geography, University of Florida, Gainesville, FL}

